\title{
Collaborative Decision-Making in Product Design: An Interactive Multiobjective Approach
}

\author{
Chantal Baril, ${ }^{1}$ Soumaya Yacout, ${ }^{2}$ and Bernard Clément ${ }^{2}$ \\ ${ }^{1}$ Department of Industrial Engineering, Université du Québec à Trois-Rivières, 3351 Boulevard des Forges, Trois-Rivières, \\ QC, Canada G9A 5 H7 \\ ${ }^{2}$ Department of Mathematical and Industrial Engineering, École Polytechnique de Montréal, 2900 Boulevard \\ Édouard-Montpetit, Campus de l'Université de Montréal, Montréal, QC, Canada H3T 1J4
}

Correspondence should be addressed to Chantal Baril; chantal.baril@uqtr.ca

Received 16 August 2012; Accepted 29 October 2012

Academic Editor: C. K. Kwong

Copyright (C) 2013 Chantal Baril et al. This is an open access article distributed under the Creative Commons Attribution License, which permits unrestricted use, distribution, and reproduction in any medium, provided the original work is properly cited.

This paper presents a new procedure to solve multiobjective problems, where the objectives are distributed to various working groups and the decision process is centralized. The approach is interactive and considers the preferences of the working groups. It is based on two techniques: an interactive technique that solves multi-objective problems based on goal programming, and a technique called "linear physical programming" which considers the preferences of the working groups. The approach generates Pareto-optimal solutions. It guides the director in the determination of target values for the objective functions. The approach was tested on two problems that present its capacity to generate Pareto-optimal solutions and to show the convergence to compromise solutions for all the working groups.

\section{Introduction}

The process of product design is often organized in a hierarchical structure where the specialists are separated by discipline in several working groups. As shown in Figure 1, the working groups are supervised by a director who coordinates the design activities. The role of the director is to collect information provided by the groups and to use computational method to finding an optimal design. The working groups are considered as experts that have the technical knowledge in their proper discipline.

According to their competencies, each working group is responsible of achieving specific design objectives expressing the customer's requirements. Often these objectives are functions of the same set of design variables and in certain cases, they may be conflicting. For that reason, it is necessary to find an optimization procedure that takes into consideration that knowledge and includes it in the solution.

In this paper, we develop a new Interactive Multiobjective approach taking into account the working group's Preferences
(IMOP). The original contributions of the IMOP algorithm are the as follows.

(i) It has the ability to define a reduced set of target values that can be divided into degrees of desirability to capture the working groups' preferences. This is an important contribution because it is a challenging issue in multi-objective optimization.

(ii) It generates Pareto-optimal solutions corresponding to the working groups' preferences.

(iii) It subtracts the stability set from the reduced set of target values at each iteration, thus ensuring a different Pareto-optimal solution each time.

The proposed approach is particularly interesting when the decision process is centralized and involves many working groups who are collaborating in order to find a best compromise solution. 


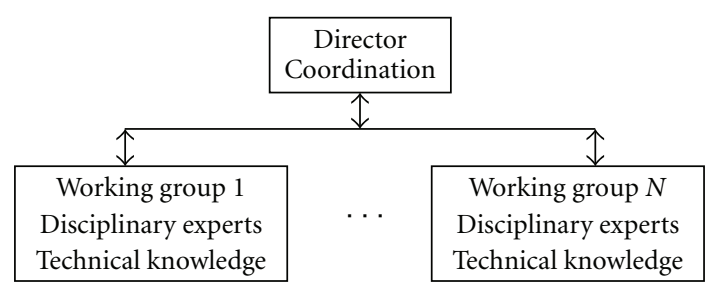

FIGURE 1: Organizational structure in product design.

\section{Multiobjective Problem and Pareto-Optimal Concept}

The purpose of a general multi-objective optimization problem is to find the design variables that optimize a vector objective function $F(X)=\left\{f_{1}(X), f_{2}(X), \ldots, f_{k}(X)\right\}$ over the feasible design space. The minimization problem formulation in standard form is as follows [1]:

$$
\begin{array}{ll}
\text { Minimize } & F(X)=\left\{f_{1}(X), f_{2}(X), \ldots, f_{k}(X)\right\} \\
\text { subject to } & h_{s}(X)=0, \quad s=1, \ldots, t, \\
& g_{r}(X) \geq 0, \quad r=1, \ldots, m, \\
& X^{l} \leq X \leq X^{u} .
\end{array}
$$

The aim of solving a multi-objective problem is to get a Pareto-optimal solution or a set of Pareto-optimal solutions. Conceptually, a Pareto-optimal solution is one which is not dominated by any other feasible solution. Mathematically, for a minimization problem with $k$ objective functions $f_{i}, i=$ $1, \ldots, k$, a vector $X^{*}$ is Pareto-optimal if there is no other feasible $X$ such that $f(X) \leq f\left(X^{*}\right)$, meaning that $f_{i}(X) \leq$ $f_{i}\left(X^{*}\right)$ for all $i=1, \ldots, k$ with strict inequality for at least one $i$ [2]. In general, the optimal solutions obtained by the individual optimization of the objectives are not the same. It is then necessary to find solutions to the multi-objective problem which are Pareto-optimal.

There are several techniques for solving multi-objective optimization problems. Some methods have been developed to find an exact Pareto set, or an approximation of it, inside of which one of the generated Pareto optimal solutions is chosen for implementation. These methods include compromise programming [3], the weight method, and the constraints method [4]. Several metaheuristics approaches have also been used to solve multi-objective problems like simulated annealing [5, 6] particles swarm optimization [7] and evolutionary algorithms [8-10].

However, as the number of competing objectives increases, the problem of finding the best compromise solution becomes increasingly complex. Hence, it can become overwhelming to analyze the entire Pareto-optimal solution set to select one solution for implementation. It becomes attractive to reduce the size of the solution set, and to assist the decision maker in selecting a final solution [11]. Some methods attempt to quantify the decision maker's preferences, and with this information, the solution that best satisfies the decision maker's preferences is then identified. These methods include among others goal programming, and linear physical programming [12]. Linear physical programming is a method for generating a preferred Pareto solution during multi-objective optimization. It is an extension of goal programming. The initial development of the physical programming methodology is presented in Messac et al. [12]. Physical programming captures the decision maker's preferences, a priori, in a mathematically consistent manner using a preference function. The decision maker (DM) classifies each objective function into the four soft and the four hard classes as shown in Table 1.

The DM specifies the degrees of desirability $\left(t_{i 5}^{-}, t_{i 4}^{-}, t_{i 3}^{-}\right.$, $t_{i 2}^{-}, t_{i 1}^{-}$and/or $\left.t_{i 1}^{+}, t_{i 2}^{+}, t_{i 3}^{+}, t_{i 4}^{+}, t_{i 5}^{+}\right)$for each objective function $f_{i}$ in the soft category. For classes $1 \mathrm{~S}$ through $4 \mathrm{~S}$, there are, respectively, five, nine, and ten such values as shown in Tables $2,3,4$, and 5 .

For classes $1 \mathrm{H}$ through $4 \mathrm{H}$, these values are, respectively, $t_{l \text { max }}, t_{l, \text { min }}, t_{l, \mathrm{val}}$, and $t_{l, \text { min }}$ and $t_{l \text { max }}$. The physical programming method involves converting a multi-objective problem into a single objective problem by using preference functions that capture the DM's preferences. Given the DM's input in the form of range boundaries (or targets) for each objective, Messac et al. [12] suggest an algorithm to generate the weights $\widetilde{w}_{i s}^{-}$and $\widetilde{w}_{i s}^{+}$. The following problem is then solved $\left(d_{i s}^{-}\right.$and $d_{i s}^{+}$ are the deviational variables):

$$
\begin{aligned}
& \operatorname{Min}_{d_{i s}^{-}, d_{i s}^{+}, X} \quad J=\sum_{i=1}^{k} \sum_{s=2}^{5}\left(\widetilde{w}_{i s}^{-} d_{i s}^{-}+\widetilde{w}_{i s}^{+} d_{i s}^{+}\right) \\
& \text {subject to } f(X)_{i}-d_{i s}^{+} \leq t_{i(s-1)}^{+} \\
& \forall i \in \text { classes } 1 S \text {, 3S, } 4 \text { S; } i=1,2, \ldots, k \text {; } \\
& s=2, \ldots, 5 \text {, } \\
& d_{i s}^{+} \geq 0 \quad \forall i \in \text { classes } 1 \mathrm{~S}, 3 \mathrm{~S}, 4 \mathrm{~S} \text {; } \\
& i=1,2, \ldots, k ; s=2, \ldots, 5 \\
& f_{i}(X) \leq t_{i 5}^{+} \quad \forall i \in \text { classes } 1 \text { S, 3S, } 4 \text { S } \\
& i=1,2, \ldots, k \text {, } \\
& f_{i}(X)+d_{i s}^{-} \geq t_{i(s-1)}^{-}, \quad \forall i \in \text { classes } 2 S, 3 S, 4 S \text {; } \\
& i=1,2, \ldots, k ; s=2, \ldots, 5, \\
& d_{i s}^{-} \geq 0, \quad \forall i \in \text { classes } 2 \mathrm{~S}, 3 \mathrm{~S}, 4 \mathrm{~S} \text {; } \\
& i=1,2, \ldots, k ; s=2, \ldots, 5, \\
& f_{i}(X) \geq t_{i 5}^{-}, \quad \forall i \in \text { classes } 2 \text { S, } 3 \mathrm{~S}, 4 \mathrm{~S} \text {; } \\
& i=1,2, \ldots, k \text {, } \\
& f_{l}(X) \leq t_{l, \text { max }}, \quad \forall l \in \text { class } 1 H \text {; } \\
& l=1,2, \ldots, L, \\
& f_{l}(X) \geq t_{l, \min }, \quad \forall l \in \text { class } 2 H ; \\
& l=1,2, \ldots, L, \\
& f_{l}(X)=t_{l, \mathrm{val}}, \quad \forall l \in \text { class } 3 H \text {; } \\
& l=1,2, \ldots, L,
\end{aligned}
$$




$$
\begin{array}{ll}
t_{l, \text { min }} \leq f_{l}(X) \leq t_{l, \max }, & \forall l \in \text { class } 4 H \\
& l=1,2, \ldots, L, \\
X_{\min } \leq X \leq X_{\max } . &
\end{array}
$$

The limitation of the physical programming is that it requires a priori selection of range parameters for each of the objective functions and provides information for only one design scenario (i.e., a single Pareto solution). Tappeta et al. [13] have twinned linear physical programming with an interactive algorithm [1]. Their algorithm finds a Pareto solution and can generate other Pareto designs in the neighbourhood of the current Pareto solution. No means are provided to help the DM to specify his/her initial preferences in the form of region limits defined in physical programming.

Some authors have suggested several interactive multiobjective optimization methods [1,14-16]. These methods allow the decision maker to express his/her preferences by using a reference point or by classifying the objectives, functions. The disadvantages of using traditional multi-objective methods are as follows [1]: (1) require a priori selection of weights or targets for each of the objective functions, (2) provide only a single Pareto-optimal solution, and (3) are unable to generate proper Pareto-optimal points for non convex problems (the weights method). Abdel Haleem [17] developed an interactive nonlinear goal programming algorithm (INLGP) that helps the decision maker to determine reference points for the goals. The decision maker does not need to do any ranking of classification of these goals. The advantages of this INLGP algorithm are as follows: (1) it reduces the parametric space of the target values by limiting each parameter with minimum and maximum values rather than by choosing any random values from the whole parametric space, and (2) the algorithm is guaranteed to generate Pareto-optimal solutions at each iteration. The INLGP algorithm was used for the design of a low-pass electrical circuit [18]. However, no means are provided to divide the reduced parametric space. Realizing these limitations, an interactive multi-objective approach is proposed which attempts to address the issues mentioned above.

\section{An Interactive Multiobjective Approach Taking into Account the Working Groups' Preferences (IMOP Approach)}

The IMOP approach is based on the interactive nonlinear goal programming algorithm (INLGP) of Abdel Haleem [17] combined to the linear physical programming introduced by Messac et al. [12]. The IMOP approach has the following advantages: (1) it provides means to capture the working group's preferences, (2) it offers the possibility of interaction between the director and his/her working groups, (3) it generates several Pareto-optimal solutions (several design scenarios), and (4) it fits with the industries organizational structure.

Before using the IMOP approach, it is necessary to

\begin{tabular}{|c|c|c|}
\hline & Class $1 \mathrm{~S}$ & Small is better (minimization) \\
\hline \multirow[t]{4}{*}{ Soft } & Class $2 \mathrm{~S}$ & Larger is better (maximization) \\
\hline & Class 3S & Value is better \\
\hline & Class $4 \mathrm{~S}$ & Range is better \\
\hline & Class $1 \mathrm{H}$ & Must be smaller $\left(f_{l} \leq t_{l, \max }\right)$ \\
\hline \multirow[t]{3}{*}{ Hard } & Class $2 \mathrm{H}$ & Must be larger $\left(f_{l} \geq t_{l, \min }\right)$ \\
\hline & Class $3 \mathrm{H}$ & Must be equal $\left(f_{l}=t_{l, \mathrm{val}}\right)$ \\
\hline & Class $4 \mathrm{H}$ & $\begin{array}{c}\text { Must be in range } \\
\left(t_{l, \min } \leq f_{l} \leq t_{l, \max }\right)\end{array}$ \\
\hline
\end{tabular}
distribute the objective functions among the working groups
TABLE 1: Objective function classification.

according to their respective disciplinary competencies. More than one objective can be assigned to the same working group. The multi-objective optimization process is centralized at the director level. The director coordinates the activities between the working groups. The working groups collaborate to the resolution process by defining their preferences and providing the target values for their objective functions. The following are the steps involved in the application of the new interactive multi-objective approach.

Step 1. Each working group classifies his objective functions into four classes (Table 6).

Step 2. For the $k$ objective functions, each working group solves its objective optimization problem individually according to a category chosen in 1 . The optimal solutions are $X^{* i}, i=1, \ldots, k$. The optimal values of the objective functions are $f_{i}^{*}, i=1, \ldots, k$. The working groups know the best possible values of each objective function under their control. These values are returned to the director.

Step 3. The director evaluates the value of the other $k-1$ objective functions at the $k$ optimal solutions $X^{* i}, i=1, \ldots, k$, and constructs $k \times k$ table of the objectives values as shown in Table 7. From this table, the director will know the best and the worst possible values of each objective function $f_{i}^{*}, i=$ $1, \ldots, k$ that corresponding to $b_{i \min }, b_{i \max }, i=1, \ldots, k$ of each objective function (for a minimization problem). The approach proceeds by determining the reduced solvability set denoted by $D^{\prime}$ where $D^{\prime}=\left\{D \mid b_{i \min } \leq b_{i} \leq b_{i \max }, i=\right.$ $1, \ldots, k\}$ and $D$ is the set of parameter values for which the problem is solvable. The reduced solvability set will be used by the working groups to define their preference according to their competencies.

Step 4 . The director presents the reduced solvability set $D^{\prime}$ to the working groups to seek preferences for each objective function.

(i) For class 1S (minimization), the preferences are highly desirable $\left(t_{i 1}^{+}\right)$, desirable $\left(t_{i 2}^{+}\right)$, tolerable $\left(t_{i 3}^{+}\right)$, undesirable, and $\left(t_{i 4}^{+}\right)$and highly undesirable $\left(t_{i 5}^{+}\right)$.

(ii) For class $2 \mathrm{~S}$ (maximization), the preferences are highly desirable $\left(t_{i 1}^{-}\right)$, desirable $\left(t_{i 2}^{-}\right)$, tolerable $\left(t_{i 3}^{-}\right)$, undesirable $\left(t_{i 4}^{-}\right)$, and highly undesirable $\left(t_{i 5}^{-}\right)$. 
(iii) For class $3 \mathrm{~S}$ (value is better), the preferences are highly desirable $\left(t_{i 1}\right)$, desirable $\left(t_{i 2}^{-}\right.$and $\left.t_{i 2}^{+}\right)$, tolerable $\left(t_{i 3}^{-}\right.$and $\left.t_{i 3}^{+}\right)$, undesirable $\left(t_{i 4}^{-}\right.$and $\left.t_{i 4}^{+}\right)$and highly undesirable $\left(t_{i 5}^{-}\right.$and $\left.t_{i 5}^{+}\right)$.

(iv) For class $4 \mathrm{~S}$ (range is better), the preferences are highly desirable $\left(t_{i 1}^{-}\right.$and $\left.t_{i 1}^{+}\right)$, desirable $\left(t_{i 2}^{-}\right.$and $\left.t_{i 2}^{+}\right)$, tolerable $\left(t_{i 3}^{-}\right.$and $\left.t_{i 3}^{+}\right)$, undesirable $\left(t_{i 4}^{-}\right.$and $\left.t_{i 4}^{+}\right)$, and highly undesirable $\left(t_{i 5}^{-}\right.$and $\left.t_{i 5}^{+}\right)$.

For multi-objective problem, the director has not all the necessary competencies to choose these values. It is why the collaboration of the working groups is important. For example, the following scenario can be used to define the degrees of desirability for a pure mathematical minimization problem. Supposing that $t_{i 1}^{+}=b_{i \mathrm{Min}}, t_{i 5}^{+}=b_{i \mathrm{Max}}$ and $\left(b_{i \mathrm{Max}}-\right.$ $\left.b_{i \text { Min }}\right) / 4=v_{i}$, the reduced solvability set $D^{\prime}$ can be divided as follows: $t_{i 1}^{+}=b_{i \mathrm{Min}}, t_{i 2}^{+}=t_{i 1}^{+}+v_{i}, t_{i 3}^{+}=t_{i 2}^{+}+v_{i}, t_{i 4}^{+}=$ $t_{i 3}^{+}+v_{i}$, and $t_{i 5}^{+}=b_{i \mathrm{Max}}$. For design problem, these values are set according to the working groups' competencies and customer's requirements.

Step 5. Set solution $j=1$. Each working group selects the target value $b_{i}$ for each of their objective functions and transfers these values to the director.

Step 6. The director uses the algorithm proposed by Dauer and Krueger [19] to solve the following multiobjective goal programming problem and to obtain the Pareto-optimal solution $\bar{X}_{j}$. This algorithm is detailed in Appendix A. The last attainment problem for goal $k$ twinned with the linear physical programming is $\left(P_{k}\right)$

Minimize $d_{k}$

subject to $M(b)=\left\{X \in R^{n} \mid g_{r}(X) \leq b_{r}, r=1, \ldots, m, X \geq 0\right\}$

and for classes $1 \mathrm{~S}, 3 \mathrm{~S}$, and $4 \mathrm{~S}$

$$
\begin{gathered}
g_{m+i}(X) \equiv f_{i}(X)-d_{i} \leq b_{i}, \quad 1 \leq i \leq k, \\
d_{i}=d_{i}^{*}, \quad 1 \leq i \leq k-1, \\
f_{i}(X) \leq t_{i 5}^{+}, \quad 1 \leq i \leq k, \\
d_{k} \geq 0
\end{gathered}
$$

and for classes 2 S, 3S, and 4 S

$$
\begin{gathered}
g_{m+i}(X) \equiv f_{i}(X)+d_{i} \geq b_{i}, \quad 1 \leq i \leq k, \\
d_{i}=d_{i}^{*}, \quad 1 \leq i \leq k-1, \\
f_{i}(X) \geq t_{i 5}^{-}, \quad 1 \leq i \leq k, \\
d_{k} \geq 0 .
\end{gathered}
$$

For all classes

$$
X_{\min } \leq X \leq X_{\max }
$$

Note. The constraints $g_{m+i}(X)$ are called the goals constraints.
This step permits to find a solution that meets as much as possible the working group's preferences.

Step 7. If the working groups are satisfied with this solution, stop and go to Step 13, if not, go to Step 8. It is suggested to generate a certain number of optimal solutions, which are Pareto optimal before stopping.

Step 8. The director formulates the KKT conditions of the problem $\left(P_{k}\right)$ and determines the values of the Kuhn Tucker multipliers associated with the goals constraints: $\bar{u}_{r}, r=1$, $\ldots, k+m$.

Step 9. According to the values $\bar{u}_{r}$ and by using the algorithm presented in Appendix B, the director determines the stability set of the first kind $G\left(\bar{X}_{j}\right)$ which is the set of parameter values for which the optimal solution remains optimal.

Step 10. The director uses the sets subtraction algorithm presented in Appendix $\mathrm{C}$ to obtain the new reduced solvability set $\left\{D^{\prime}-\bigcup_{p=1}^{j} G\left(\bar{X}_{p}\right)\right\}$ which excludes the stability set. Steps 8 , 9 , and 10 are necessary to ensure that the work groups will choose target values leading to other Pareto-optimal solution.

Step 11. If no values can be chosen in $\left\{D^{\prime}-\bigcup_{p=1}^{j} G\left(\bar{X}_{p}\right)\right\}$, stop and go to Step 13, otherwise go to Step 12.

Step 12. Set $j=j+1$. The working groups select other target values $b_{i} \in\left\{D^{\prime}-\bigcup_{p=1}^{j-1} G\left(\bar{X}_{p}\right)\right\}$ and go to Step 6. One can use these rules to select the values and to obtain other Pareto optimal solutions.

(i) Rule no. 1: It is always necessary to improve the objective function having the worst value by choosing its target value in a better zone and by sacrificing the other objectives by choosing their target values in a less desirable zone. The aim of these choices is to obtain, if possible, all the objective's values in the tolerable zone (or better).

(ii) Rule no. 2: Once in the tolerable zone, try other values in this zone in order to obtain other Pareto-optimal solutions. The selected values should cover all the zone. For example, choose a value at one end of the tolerable zone and the other values at the other end. One can also try to choose one of the target values in the desirable zone while leaving the other target values in the tolerable zone.

(iii) Rule no. 3: if it is impossible to follow the first rule due to the reduced solvability set, try all the possibilities to find the best choice.

Step 13. The director presents all the Pareto-optimal solutions to the working groups and tries to get consensus for the best compromise. If other solutions are necessary, go to Step 12. 
TABLE 2: Degrees of desirability for class $1 \mathrm{~S}$.

\begin{tabular}{lcccc}
\hline \multicolumn{4}{c}{ Class 1S-smaller is better (i.e., minimization) } \\
\hline$f_{i} \leq t_{i 1}^{+}$ & $t_{i 1}^{+}<f_{i} \leq t_{i 2}^{+}$ & $t_{i 2}^{+}<f_{i} \leq t_{i 3}^{+}$ & $t_{i 3}^{+}<f_{i} \leq t_{i 4}^{+}$ & $t_{i 4}^{+}<f_{i} \leq t_{i 5}^{+}$ \\
Highly desirable & Desirable & Tolerable & Undesirable & Highly undesirable \\
\hline
\end{tabular}

TABLE 3: Degrees of desirability for class 2 S.

\begin{tabular}{lcccc}
\hline \multicolumn{4}{c}{ Class 2S-larger is better (i.e., maximization) } \\
\hline$t_{i 5}^{-} \leq f_{i}<t_{i 4}^{-}$ & $t_{i 4}^{-} \leq f_{i}<t_{i 3}^{-}$ & $t_{i 3}^{-} \leq f_{i}<t_{i 2}^{-}$ & $t_{i 2}^{-} \leq f_{i}<t_{i 1}^{-}$ & $t_{i 1}^{-} \leq f_{i}$ \\
Highly undesirable & Undesirable & Tolerable & Desirable & Highly desirable \\
\hline
\end{tabular}

TABLE 4: Degrees of desirability for class 3S.

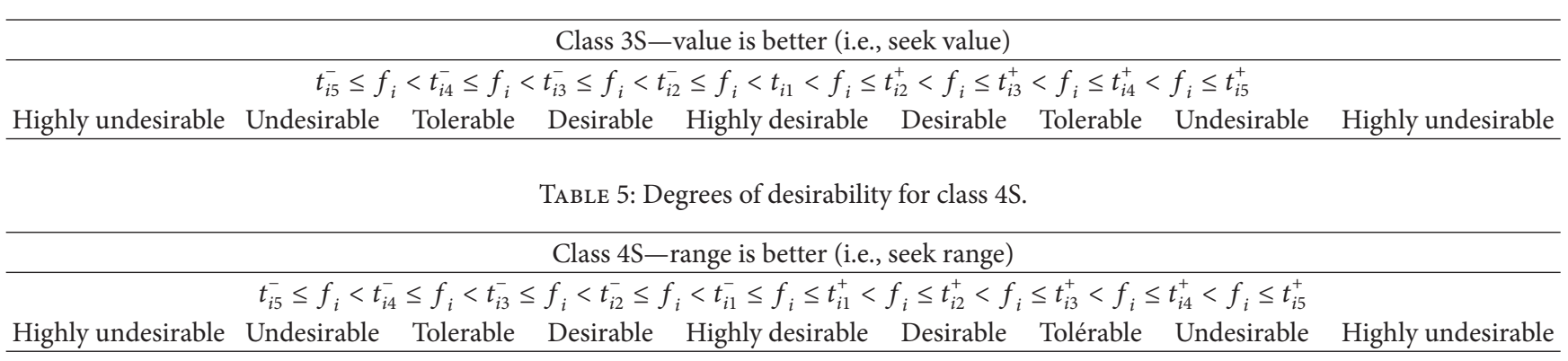

TABLE 6: Classification of the objective functions.

\begin{tabular}{lc}
\hline Class $1 S$ & Small is better (minimization) \\
Class $2 \mathrm{~S}$ & Larger is better (maximization) \\
Class $3 \mathrm{~S}$ & Value is better (seek value) \\
Class $4 \mathrm{~S}$ & Range is better (seek range) \\
\hline
\end{tabular}

TABLE 7: Table of objectives values.

\begin{tabular}{lcccc}
\hline Optimal solutions & \multicolumn{4}{l}{ Objective functions } \\
\hline$X^{* 1}$ & $f_{1}^{*}\left(X^{* 1}\right)$ & $f_{2}^{*}\left(X^{* 1}\right)$ & $\ldots$ & $f_{i}^{*}\left(X^{* 1}\right)$ \\
$X^{* 2}$ & $f_{1}^{*}\left(X^{* 2}\right)$ & $f_{2}^{*}\left(X^{* 2}\right)$ & $\ldots$ & $f_{i}^{*}\left(X^{* 2}\right)$ \\
$\ldots$ & $\ldots$ & $\ldots$ & $\ldots$ & $\ldots$ \\
$X^{* i}$ & $f_{1}^{*}\left(X^{* i}\right)$ & $f_{2}^{*}\left(X^{* i}\right)$ & $\ldots$ & $f_{i}^{*}\left(X^{* i}\right)$ \\
\hline
\end{tabular}

\section{Numerical Examples}

In this section, the interactive multi-objective procedure is applied to two design problems. The first problem consists of a set of simple analytical expressions for its objective and constraint functions and was presented by Tappeta et al. [13]. This problem is chosen to illustrate the key features of the approach and to compare with the results obtained by those authors. The second problem is the design of a twobar structure that is subjected to a force, $F$, at a point that vertically deflects by an amount $d$. In both cases, the IMOP approach is implemented in Matlab 7.0.4.365 (R14) and the optimization process was conducted on Pentium D duo core 3.4 GHz and $2 \mathrm{~GB}$ RAM. The computational time is less than 1 minute.
4.1. Test Problem 1. This problem was introduced by Tappeta et al. [13] and has three design variables, three objective functions, and a constraint. The problem definition in standard form and the application of the IMOP approach are as follows:

$$
\begin{array}{cc}
\text { Minimize } & F(X)=\left\{f_{1}(X), f_{2}(X), f_{3}(X)\right\} \\
\text { subject to } & g_{1}(X)=12-x_{1}^{2}-x_{2}^{2}, \\
X \geq 0,
\end{array}
$$

where

$$
\begin{aligned}
& f_{1}(X)=10-\frac{\left(x_{1}^{3}+x_{1}^{2}\left(1+x_{2}+x_{3}\right)+x_{2}^{3}+x_{3}^{3}\right)}{10}, \\
& f_{2}(X)=15-\frac{\left(x_{1}^{3}+2 x_{2}^{3}+x_{2}^{2}\left(2+x_{1}+x_{3}\right)+x_{3}^{3}\right)}{10}, \\
& f_{3}(X)=20-\frac{\left(x_{1}^{3}+x_{2}^{3}+3 x_{3}^{3}+x_{3}^{2}\left(3+x_{1}+x_{2}\right)\right)}{10} .
\end{aligned}
$$

For this example, we suppose that $f_{1}(X)$ and $f_{2}(X)$ needed specific competencies so they are assigned to a working group and $f_{3}(X)$ need other competencies so it is assigned to another group. Therefore, the procedure proceeds with a director and two working groups.

Step 1. Each working group classifies its objective functions:

Working group 1 classifies $f_{1}(X)$ in class $1 \mathrm{~S}$,

Working group 1 classifies $f_{2}(X)$ in class $1 \mathrm{~S}$,

Working group 2 classifies $f_{3}(X)$ in class 1 S.

Step 2. For the $k$ objective functions, each working group solves its single optimization problem individually according 
TABLE 8: Optimal values for the objective functions of test Problem 1.

\begin{tabular}{lcccc}
\hline$X^{* i}$ & $x_{1}$ & $x_{2}$ & $x_{3}$ & $f_{i}^{*}$ \\
\hline Working group 1 & 3.2539 & 0.8402 & 0.8402 & 3.5980 \\
Working group 1 & 0.4651 & 3.4011 & 0.4651 & 3.7221 \\
Working group 2 & 0.3169 & 0.3169 & 3.4350 & 3.5471 \\
\hline
\end{tabular}

to the category chosen in 1 . The optimal solutions are $X^{* i}, i=1, \ldots, k$. The optimal values of the objective functions are noted to be $f_{i}^{*}, i=1, \ldots, k$ and are presented in Table 8.

Step 3. The director evaluates the value of the other $k-1$ objective function at the $k$ optimal solutions and constructs the $k \times k$ table of the objective values. From Table 9 , the director knows the best and the worst values for each objective function. These values are noted to be $b_{i \text { min }}, b_{i \text { max }}, i=$ $1, \ldots, k$. The approach proceeds by determining the reduced solvability set $D^{\prime}=\left\{\{D\} \mid b_{i \min } \leq b_{i} \leq b_{i \max }, i=1, \ldots, k\right\}$ where $D$ is the set of parameters for which the problem is solvable.

The reduced solvability set is

$$
\begin{aligned}
& 3.5980 \leq b_{1} \leq 5.9405 \\
& 3.7221 \leq b_{2} \leq 10.9465 \\
& 3.5471 \leq b_{3} \leq 15.8771
\end{aligned}
$$

Step 4. The director presents the reduced solvability set $D^{\prime}$ to the working groups to seek their preferences for each objective function. These values are set according to the working groups' knowledge and experience. For class 1S, each working group determines the degrees of desirability $t_{i 1}^{+}, t_{i 2}^{+}, t_{i 3}^{+}, t_{i 4}^{+}, t_{i 5}^{+}$. Table 10 shows the degrees of desirability fixed by Tappeta et al. [13]. These degrees of desirability are used to be able to compare the results.

It is obvious that the degrees of desirability $t_{i 2}^{+}$for the objective functions $f_{1}$ and $f_{2}$ could never be reached, since they are not included in the reduced solvability set: the minimal value for the objective function $f_{1}$ is 3.5980 and for $f_{2}$ is 3.7221. This example shows that the degrees of desirability should not be given blindly to prevent the choice of scenarios which are not feasible. Table 11 shows more realistic degrees of desirability. These degrees of desirability are obtained by dividing the solvability set $3.5980 \leq b_{1} \leq$ $5.9405,3.7221 \leq b_{2} \leq 10.9465$ and $3.5471 \leq b_{3} \leq 15.8771$ according to this scenario: we suppose that the worst value is undesirable $\left(t_{i 4}^{+}=b_{i \text { Max }}\right)$ and we calculate $\left(b_{i \operatorname{Max}}-b_{i \text { Min }}\right) / 4=$ $v_{i}$ to find the following degrees of desirability: $t_{i 1}^{+}=t_{i 2}^{+}-v_{i}$, $t_{i 2}^{+}=t_{i 3}^{+}-v_{i}, t_{i 3}^{+}=t_{i 4}^{+}-v_{i}, t_{i 4}^{+}=b_{i \mathrm{Max}}$, and $t_{i 5}^{+}=t_{i 4}^{+}+v_{i}$.

We assume that preferences are uniformly distributed across the solvability set but it is not necessarily always the case.

Step 5. Set solution $j=1$. The working groups select the target values $b_{i}$ for each objective function. It is obvious that
TABLE 9: Objective function values table for test Problem 1.

\begin{tabular}{|c|c|c|c|c|c|c|}
\hline Criteria & Class & $\begin{array}{c}\mathrm{HD} \\
t_{i 1}^{+}\end{array}$ & $\begin{array}{c}\mathrm{D} \\
t_{i 2}^{+}\end{array}$ & $\begin{array}{r}\mathrm{T} \\
t_{i 3}^{+}\end{array}$ & $\begin{array}{c}\mathrm{U} \\
t_{i 4}^{+}\end{array}$ & $\begin{array}{c}\mathrm{HU} \\
t_{i 5}^{+}\end{array}$ \\
\hline$f_{1}$ & $1 \mathrm{~S}$ & 3.0 & 4.25 & 6.0 & 7.5 & 9.0 \\
\hline$f_{2}$ & $1 \mathrm{~S}$ & 3.7 & 7.0 & 9.25 & 11.8 & 12.5 \\
\hline$f_{3}$ & $1 S$ & 6.0 & 12.0 & 15.0 & 18.0 & 20.0 \\
\hline
\end{tabular}

\begin{tabular}{ccccccc}
\hline & $x_{1}$ & $x_{2}$ & $x_{3}$ & $f_{1}$ & $f_{2}$ & $f_{3}$ \\
\hline$X^{* 1}$ & 3.2539 & 0.8402 & 0.8402 & 3.5980 & 10.9465 & 15.8166 \\
$X^{* 2}$ & 0.4651 & 3.4011 & 0.4651 & 5.9405 & 3.7221 & 15.8771 \\
$X^{* 3}$ & 0.3169 & 0.3169 & 3.4350 & 5.8929 & 10.8797 & 3.5471 \\
\hline
\end{tabular}

TABLE 10: The degrees of desirability specified by Tappeta et al. [13].

HD: (highly desirable $\left.\leq t_{i 1}^{+}\right)$, D: $\left(t_{i 1}^{+}<\right.$desirable $\left.\leq t_{i 2}^{+}\right)$, T: $\left(t_{i 2}^{+}<\right.$tolerable $\leq$ $\left.t_{i 3}^{+}\right)$, ID: $\left(t_{i 3}^{+}<\right.$undesirable $\left.\leq t_{i 4}^{+}\right)$, IA: $\left(t_{i 4}^{+}<\right.$highly undesirable $\left.\leq t_{i 5}^{+}\right)$.

TABLE 11: The working group's preferences for test Problem 1.

\begin{tabular}{lcccccc}
\hline Criteria & \multirow{2}{*}{ Class } & I & D & T & ID & IA \\
& & $t_{i 1}^{+}$ & $t_{i 2}^{+}$ & $t_{i 3}^{+}$ & $t_{i 4}^{+}$ & $t_{i 5}^{+}$ \\
\hline$f_{1}$ & $1 S$ & 4.1836 & 4.7693 & 5.3549 & 5.9405 & 6.5261 \\
$f_{2}$ & $1 S$ & 5.5282 & 7.3343 & 9.1404 & 10.9465 & 12.7526 \\
$f_{3}$ & $1 S$ & 6.6296 & 9.7121 & 12.7946 & 15.8771 & 18.9596 \\
\hline
\end{tabular}

HD: (highly desirable $\left.\leq t_{i 1}^{+}\right)$, D: $\left(t_{i 1}^{+}<\right.$desirable $\left.\leq t_{i 2}^{+}\right)$, T: $\left(t_{i 2}^{+}<\right.$tolerable $\leq$ $\left.t_{i 3}^{+}\right)$, ID: $\left(t_{i 3}^{+}<\right.$undesirable $\left.\leq t_{i 4}^{+}\right)$, IA: $\left(t_{i 4}^{+}<\right.$highly undesirable $\left.\leq t_{i 5}^{+}\right)$.

each working group wants to obtain the better value for their objective functions. So they will choose target values in the highly desirable zone. We assume that the approach starts with the target values corresponding to $t_{i 1}^{+}$:

Working group 1 sets the target value of $b_{1}$ at 4.1836 (highly desirable),

Working group 2 sets the target value of $b_{2}$ at 5.5282 (highly desirable),

Working group 3 sets the target value of $b_{3}$ at 6.6296 (highly desirable).

Step 6. With the target values supplied by the working groups, the director uses the algorithm proposed by Dauer and Krueger [19] given in Appendix A to solve the multiobjective goal programming problem and to obtain a first Pareto optimal solution $\bar{X}_{1}$ :

$$
\begin{aligned}
& \bar{X}_{1}=(2.8568,1.8775,0.5598), \\
& f_{1}=4.1836 \text { (the value of } f_{1} \text { is in the highly desirable } \\
& \text { zone), } \\
& f_{2}=9.4178 \text { (the value of } f_{2} \text { is in the undesirable } \\
& \text { zone), } \\
& f_{3}=16.7115 \text { (the value of } f_{3} \text { is in the highly } \\
& \text { undesirable zone). }
\end{aligned}
$$

Step 7. If the working groups are satisfied with this solution, stop and go to Step 13, if not, go to Step 8. For this case, 
we assume that the working groups 1 and 2 are not satisfied since the values of their objective functions $f_{2}$ and $f_{3}$ are in the undesirable and highly undesirable zones, respectively, and want to generate another solution. Go to Step 8. Steps 8, 9 , and 10 are necessary to ensure that the work groups will choose target values leading to other Pareto-optimal solution.

Step 8. The director formulates the KKT conditions of the problem and determines the values of the Kuhn Tucker multipliers associated with the goal constraints $\bar{u}_{r}, r=1, \ldots$, $k+m$,

$$
\bar{u}_{2}=1.2771 \times 10^{5}, \quad \bar{u}_{3}=5.6776 \times 10^{4}, \quad \bar{u}_{4}=1 .
$$

Step 9. According to the values $\bar{u}_{r}$, and by using the algorithm of Osman [20] given in Appendix B, the director determines the stability set $G\left(\bar{X}_{1}\right)$

$$
\begin{aligned}
& \text { Given } \bar{u}_{2}>0 \text { and } g_{2}=4.1836 \text { then } b_{1}=4.1836, \\
& \text { Given } \bar{u}_{3}>0 \text { and } g_{3}=5.5282 \text { then } b_{2}=5.5282, \\
& \text { Given } \bar{u}_{4}>0 \text { and } g_{4}=6.6296 \text { then } b_{3}=6.6296 \text {. }
\end{aligned}
$$

Step 10. The director uses the sets subtraction algorithm proposed by Abdel Haleem [17] given in Appendix $\mathrm{C}$ to obtain the new reduced solvability set $\left\{D^{\prime}-\bigcup_{p=1}^{j} G\left(\bar{X}_{p}\right)\right\}$ given in Table 12.

Step 11. If no values can be selected in $\left\{D^{\prime}-\bigcup_{p=1}^{j} G\left(\bar{X}_{p}\right)\right\}$ stop and go to Step 13, otherwise go to Step 12. In this case, other values can be chosen in Table 10 so go to Step 12 .

Step 12. Set $j=j+1$. The working groups select other target values for their objective function in $b^{j} \in\left\{D^{\prime}-\bigcup_{p=1}^{j-1} G\left(\bar{X}_{p}\right)\right\}$ and go to Step 6 . The solutions obtained are presented in Table 13.

The third Pareto-optimal solution seems to be the best one because all the objective values match the target values according to Table 10. This solution can be considered satisfactory and a good compromise for all the working groups.

Step 13. The director presents the Pareto-optimal solutions obtained to the working groups to select the best one for everyone (stop). If other solutions are necessary go to Step 12. Although the solutions obtained in the six iterations are Pareto optimal, the best Pareto-optimal solutions according to the working group's preferences (desirability) are solutions 3 and 4 . These solutions can be retained for implementation.

Finally, it is also interesting to know if the solutions (Pareto points) obtained by this approach are close to certain targeted aspiration points. To do this, we compare the obtained results with the Pareto-optimal results obtained by

\begin{tabular}{|c|c|c|}
\hline Solution $j$ & Target values $b_{i}$ & $\begin{array}{c}\text { Objective function values } \\
f_{i}\end{array}$ \\
\hline \multirow{3}{*}{1} & $\begin{array}{c}b_{1}=4.1836 \\
\text { (highly desirable) }\end{array}$ & $\begin{array}{c}f_{1}=4.1836 \\
\text { (highly desirable) }\end{array}$ \\
\hline & $\begin{array}{c}b_{2}=5.5282 \\
\text { (highly desirable) }\end{array}$ & $\begin{array}{l}f_{2}=9.4178 \\
\text { (undesirable) }\end{array}$ \\
\hline & $\begin{array}{c}b_{3}=6.6296 \\
\text { (highly desirable) }\end{array}$ & $\begin{array}{c}f_{3}=16.7115 \\
\text { (highly undesirable) }\end{array}$ \\
\hline \multirow{3}{*}{2} & $b_{1}=4.5($ desirable $)$ & $f_{1}=4.5($ desirable $)$ \\
\hline & $b_{2}=8($ tolerable $)$ & $f_{2}=8.6799$ (tolerable) \\
\hline & $b_{3}=13$ (undesirable) & $\begin{array}{c}f_{3}=16.8579 \\
\text { (highly undesirable) }\end{array}$ \\
\hline \multirow{3}{*}{3} & $b_{1}=5.2($ tolerable $)$ & $f_{1}=5.2($ tolerable $)$ \\
\hline & $b_{2}=9($ tolerable $)$ & $f_{2}=9($ tolerable $)$ \\
\hline & $b_{3}=14$ (undesirable) & $f_{3}=14.8087$ (undesirable) \\
\hline \multirow{3}{*}{4} & $b_{1}=5.3($ tolerable $)$ & $f_{1}=5.3($ tolerable $)$ \\
\hline & $b_{2}=9.1$ (tolerable $)$ & $f_{2}=9.1$ (tolerable) \\
\hline & $b_{3}=12.5$ (tolerable $)$ & $f_{3}=14.4381$ (undesirable) \\
\hline \multirow{3}{*}{5} & $b_{1}=5.9$ (undesirable) & $f_{1}=5.9$ (undesirable) \\
\hline & $b_{2}=8.9($ tolerable $)$ & $f_{2}=8.9($ tolerable $)$ \\
\hline & $b_{3}=12.6$ (tolerable $)$ & $f_{3}=13.0147$ (undesirable) \\
\hline \multirow{3}{*}{6} & $b_{1}=5($ tolerable $)$ & $f_{1}=5($ tolerable $)$ \\
\hline & $b_{2}=10$ (undesirable) & $f_{2}=10$ (undesirable) \\
\hline & $b_{3}=12($ tolerable $)$ & $f_{3}=13.9899$ (undesirable) \\
\hline
\end{tabular}

TABle 12: The reduced solvability set for test Problem 1.

\begin{tabular}{lcccccc}
\hline $\begin{array}{l}\text { Set } \\
\text { number }\end{array}$ & $b_{1} \min$ & $b_{1} \max$ & $b_{2} \min$ & $b_{2} \max$ & $b_{3} \min$ & $b_{3} \max$ \\
\hline 1 & 3.5980 & 4.1836 & 3.7221 & 10.9465 & 3.5471 & 15.8771 \\
2 & 4.1836 & 5.9405 & 3.7221 & 10.9465 & 3.5471 & 15.8771 \\
3 & 4.1836 & 4.1836 & 3.7221 & 5.5282 & 3.5471 & 15.8771 \\
4 & 4.1836 & 4.1836 & 5.5282 & 10.9465 & 3.5471 & 15.8771 \\
5 & 4.1836 & 4.1836 & 5.5282 & 5.5282 & 3.5471 & 6.6296 \\
6 & 4.1836 & 4.1836 & 5.5282 & 5.5282 & 6.6296 & 15.8771 \\
\hline
\end{tabular}

TABLE 13: Pareto-optimal solutions generated by the approach.

Tappeta et al. [13] at specific aspiration points. The comparison is presented in Table 14.

For a minimization problem, we want to find a better (smaller) solution than or equal the aspiration values. The symbol ( + ) indicates that the solution obtained by our approach is worse (bigger) than the aspiration values, the symbol $(=)$ indicates that the solution obtained is the same (equal) as the aspiration values, and the symbol (-) indicates that the solution obtained is better (smaller) than the aspiration values. The solutions obtained with our algorithm are considered better than or equal to those found by Tappeta et al. [13] if the number of symbols $(-)$ and (=) exceeds the number of symbols $(+)$. For the first solution, the number of symbols $(-)$ and $(=)$ is 2 for the IMOP algorithm and the number of symbols ( $(-)$ and $(=)$ is 1 for Tappet et al. [13]. These results are very encouraging because they demonstrate that our approach can find solutions closer to the working 
TABle 14: Aspiration points and Pareto data from Tappeta et al. [13].

\begin{tabular}{|c|c|c|c|c|c|c|c|c|}
\hline \multicolumn{3}{|c|}{ Aspiration values (target values) } & \multicolumn{3}{|c|}{ Pareto points Tappeta et al. [13] } & \multicolumn{3}{|c|}{ Pareto points IMOP approach } \\
\hline 1 & 2 & 3 & 1 & 2 & 3 & 1 & 2 & 3 \\
\hline 7.483 & 6.788 & 11.285 & $6.956(-)$ & $7.437(+)$ & $11.496(+)$ & $6.9722(-)$ & $6.7880(=)$ & $12.3239(+)$ \\
\hline 5.400 & 6.788 & 16.927 & $5.413(+)$ & $6.916(+)$ & $16.218(-)$ & $4.4679(-)$ & $5.1878(-)$ & $16.3795(-)$ \\
\hline 6.016 & 10.183 & 11.285 & $3.994(-)$ & $10.095(-)$ & $16.130(+)$ & $6.0002(-)$ & $9.9646(-)$ & $10.7528(-)$ \\
\hline 3.933 & 10.183 & 16.927 & $4.708(+)$ & $8.689(-)$ & $16.259(-)$ & $3.9235(-)$ & $10.0191(-)$ & $16.4693(-)$ \\
\hline
\end{tabular}

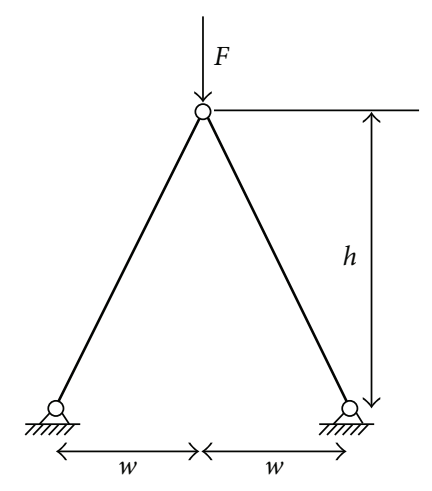

FIgURE 2: Two-bar truss example.

group's requirements (aspiration values) than algorithms available in the literature.

4.2. Test Problem 2. The second problem is the design of a two-bar structure that is subjected to a force, $F$, at a point that vertically deflects by an amount, $d$. This optimization problem involves the minimization of the mass, $m$, the normal stress, $s$, and the vertical deflection, $d$, of a two-bar truss. The design variables are the diameter of the member, $x_{1}=a$, and the height, $x_{2}=h$. Normal stress must be less than the buckling stress, as a constraint. A graphical representation of the truss is shown in Figure 2 [21]. The specific parameter values are as follows: $F=150 \mathrm{kN}, t=2.5 \mathrm{~mm}$, structure width $w=750 \mathrm{~mm}$, mass density $\rho=7.8 \times 10^{-3} \mathrm{~g} / \mathrm{mm}^{3}$, and elastic modulus $E=210000 \mathrm{~N} \cdot \mathrm{mm}^{2}$.

The problem's formulation is as follows:

$$
\begin{array}{ll}
\text { Minimize } & f_{1}(X)=m=2 \pi \rho t x_{1} \sqrt{w^{2}+x_{2}^{2}}, \\
& f_{2}(X)=s=\frac{F}{2 \pi t x_{1} x_{2}} \sqrt{w^{2}+x_{2}^{2}}, \\
& f_{3}(X)=d=\frac{F\left(w^{2}+x_{2}^{2}\right)^{3 / 2}}{2 \pi t E x_{1} x_{2}^{2}} \\
\text { subject to } \quad & g_{1}(X)=\frac{F}{2 \pi t x_{1} x_{2}} \sqrt{w^{2}+x_{2}^{2}}-\frac{1}{8} \pi^{2} E \frac{t^{2}+x_{1}^{2}}{w^{2}+x_{2}^{2}} \leq 0, \\
& 1 \leq x_{1} \leq 100, \\
& 10 \leq x_{2} \leq 1000 .
\end{array}
$$

TABle 15: Optimal values for the objective function of test Problem 2.

\begin{tabular}{lccc}
\hline$X^{* i}$ & $x_{1}$ & $x_{2}$ & $f_{i}^{*}$ \\
\hline Working group 1 & 39.2944 & 335.6810 & 3956 \\
Working group 2 & 100 & 1000 & 119.3662 \\
Working group 3 & 100 & 1000 & 0.8881 \\
\hline
\end{tabular}

TABLe 16: Objective function values table for test Problem 2.

\begin{tabular}{cccccc}
\hline & $x_{1}$ & $x_{2}$ & $f_{1}$ & $f_{2}$ & $f_{3}$ \\
\hline$X^{* 1}$ & 39.2944 & 335.6810 & 3956 & 595 & 6 \\
$X^{* 2}$ & 100 & 1000 & 15315 & 119.3662 & 0.8881 \\
$X^{* 3}$ & 100 & 1000 & 15315 & 119.3662 & 0.8881 \\
\hline
\end{tabular}

For this example, we assume that each objective function needs specific competencies so one objective function is assigned to a working group. The procedure proceeds with a director and three working groups.

Step 1. Each working group classifies its objective function:

Working group 1 classifies $f_{1}(X)$ in class $1 S$,

Working group 2 classifies $f_{2}(X)$ in class $1 \mathrm{~S}$,

Working group 3 classifies $f_{3}(X)$ in class 1 S.

Step 2. Each working group solves its single optimization problem according to the category chosen in 1 . The optimal solutions are $X^{* i}, i=1, \ldots, 3$. The optimal values of the objective functions are noted to be $f_{i}^{*}, i=1, \ldots, 3$ and are presented in Table 15.

Step 3. The director evaluates the two other objective functions at the three optimal solutions and constructs the $3 \times 3$ table of the objective functions' values. From Table 16, the director knows the best and the worst values for each objective function. These values are noted to be $b_{i \min }, b_{i \max }, i=$ $1, \ldots, 3$. The approach proceeds by determining the reduced solvability set denoted by $D^{\prime}$ where $D^{\prime}=\left\{D \mid b_{i \min } \leq b_{i} \leq\right.$ $\left.b_{i \max }, i=1, \ldots, 3\right\}$ and $D$ is the set of parameters for which the problem is solvable.

The reduced solvability set is

$$
\begin{aligned}
& 3956 \leq b_{1} \leq 15315 \\
& 119.3662 \leq b_{2} \leq 595 \\
& 0.8881 \leq b_{3} \leq 6
\end{aligned}
$$


TABLE 17: Working group's preferences for test Problem 2.

\begin{tabular}{lcccccc}
\hline \multirow{2}{*}{ Function Class } & HD & $\mathrm{D}$ & $\mathrm{T}$ & $\mathrm{U}$ & $\mathrm{HU}$ \\
& & $t_{i 1}^{+}$ & $t_{i 2}^{+}$ & $t_{i 3}^{+}$ & $t_{i 4}^{+}$ & $t_{i 5}^{+}$ \\
\hline$f_{1}$ & $1 \mathrm{~S}$ & 4450 & 4550 & 4650 & 4750 & 4850 \\
$f_{2}$ & $1 \mathrm{~S}$ & 370 & 390 & 400 & 450 & 500 \\
$f_{3}$ & $1 \mathrm{~S}$ & 2 & 2.5 & 3 & 3.5 & 4 \\
\hline
\end{tabular}

HD: (highly desirable $\left.\leq t_{i 1}^{+}\right)$, D: $\left(t_{i 1}^{+}<\right.$desirable $\left.\leq t_{i 2}^{+}\right)$, T: $\left(t_{i 2}^{+}<\right.$tolerable $\leq$ $\left.t_{i 3}^{+}\right)$, ID: $\left(t_{i 3}^{+}<\right.$undesirable $\left.\leq t_{i 4}^{+}\right)$, IA: $\left(t_{i 4}^{+}<\right.$highly undesirable $\left.\leq t_{i 5}^{+}\right)$.

Step 4. The director presents the reduced solvability set $D^{\prime}$ to the working groups to seek their preferences for each objective function. These values are set according to the working groups' knowledge and experience. For class $1 \mathrm{~S}$, each working group determines $t_{i 1}^{+}, t_{i 2}^{+}, t_{i 3}^{+}, t_{i 4}^{+}, t_{i 5}^{+}$. Table 17 shows the degrees of desirability determined by Messac and IsmailYahaya [21]. These degrees of desirability are used to be able to compare the results. These degrees of desirability are realistic because they are inside the reduced solvability set determined in Step 3.

Step 5. Set solution $j=1$. Each working group selects the target values $b_{i}$ for its objective function. We assume that the working groups will not make a compromise, and they will choose target values in the highly desirable zone. The approach starts with the target value corresponding to $t_{i 1}^{+}$:

Working group 1 sets target value $b_{1}$ at 4450 (highly desirable),

Working group 2 sets target value $b_{2}$ at 370 (highly desirable),

Working group 3 sets target value $b_{3}$ at 2 (highly desirable).

Step 6. With the target values supplied by the working groups, the director uses the algorithm proposed by Dauer and Krueger [19] given in Appendix A to solve the multiobjective goal programming problem and to obtain a first Pareto optimal solution $\bar{X}_{1}$ :

$$
\bar{X}_{1}=(37.8392599 .0083) \text {, }
$$

$f_{1}=4450$ (the value of $f_{1}$ is in the highly desirable zone),

$f_{2}=404.3889$ (the value of $f_{2}$ is in the undesirable zone),

$f_{3}=2.9618$ (the value of $f_{3}$ is in the tolerable zone).

Step 7. If the working groups are satisfied with this solution, stop and go to Step 13, if not, go to Step 8. For this problem, we assume that working group 2 is not satisfied since the value of its objective function is in the undesirable zone and wants to generate another solution. Go to Step 8. Steps 8, 9, and 10 are necessary to ensure that the working groups will choose target values leading to other Pareto-optimal solution.
TABLE 18: The reduced solvability set for test Problem 2.

\begin{tabular}{lcccccc}
\hline Set no: & $b_{1} \min$ & $b_{1} \max$ & $b_{2} \min$ & $b_{2} \max$ & $b_{3} \min$ & $b_{3} \max$ \\
\hline 1 & 3956 & 4450 & 119 & 595 & 1 & 6 \\
2 & 4450 & 15315 & 119 & 370 & 1 & 6 \\
3 & 4450 & 15315 & 370 & 595 & 1 & 2 \\
\hline
\end{tabular}

TABLe 19: Pareto-optimal solutions generated for Problem test 2.

\begin{tabular}{ccc}
\hline Solution & Target values $b_{i}$ & $\begin{array}{c}\text { Objective function } \\
\text { values } f_{i}\end{array}$ \\
\hline & $b_{1}=4450$ & $f_{1}=4450$ \\
(highly desirable) & (highly desirable) \\
1 & $b_{2}=370$ & $f_{2}=404.3889$ \\
(highly desirable) & (undesirable) \\
& $b_{3}=2$ & $f_{3}=2.9618$ \\
& (highly desirable) & $f_{1}=4600$ \\
& $b_{1}=4600$ & (tolerable) \\
\hline & (tolerable) & $f_{2}=386.1490$ \\
& $b_{2}=395$ & (desirable) \\
& $($ tolerable) & $f_{3}=2.7917$ \\
& $b_{3}=1.8$ & (tolerable) \\
\hline & (highly desirable) & $f_{1}=4565$ \\
& $b_{1}=4565$ & (tolerable) \\
& (tolerable) & $f_{2}=390.0621$ \\
$b_{2}=369$ & (tolerable) \\
& (highly desirable) & $f_{3}=2.8269$ \\
$b_{3}=2.8$ & (tolerable) \\
\hline & (tolerable) &
\end{tabular}

Step 8. The director formulates the KKT conditions for the problem and determines the values of the Kuhn Tucker multipliers associated with the goal constraints $\bar{u}_{r}, r=$ $1, \ldots, k+m$

$$
\bar{u}_{2}=0, \quad \bar{u}_{3}=0, \quad \bar{u}_{4}=0 .
$$

Step 9. According to the values $\bar{u}_{r}$, and by using the algorithm of Osman [20] given in Appendix B, the director determines the stability set $G\left(\bar{X}_{j}\right)$ :

$$
\begin{aligned}
& \text { Given } \bar{u}_{2}=0 \text { and } g_{2}=4450 \text { then } b_{1} \geq 4450, \\
& \text { Given } \bar{u}_{3}=0 \text { and } g_{3}=370 \text { then } b_{2} \geq 370, \\
& \text { Given } \bar{u}_{4}=0 \text { and } g_{4}=2 \text { then } b_{3} \geq 2 .
\end{aligned}
$$

Step 10. The director uses the sets subtraction algorithm proposed by Abdel Haleem [17] given in Appendix $C$ to obtain the reduced solvability set $\left\{D^{\prime}-\bigcup_{p=1}^{j} G\left(\bar{X}_{p}\right)\right\}$ given in Table 18. 
TABLE 20: Results' comparison.

\begin{tabular}{lcc}
\hline $\begin{array}{l}\text { Two-bar structure } \\
\text { characteristics }\end{array}$ & $\begin{array}{c}\text { Results } \\
\text { IMOP approach }\end{array}$ & $\begin{array}{c}\text { Results of Messac } \\
\text { and Ismail-Yahaya } \\
{[21]}\end{array}$ \\
\hline Diameter $\left(x_{1}\right)$ & $3.80 \mathrm{~cm}$ & $3.80 \mathrm{~cm}$ \\
Height $\left(x_{2}\right)$ & $64.2 \mathrm{~cm}$ & $63.26 \mathrm{~cm}$ \\
Mass $\left(f_{1}\right)$ & $4.600 \mathrm{~kg}$ & $4.565 \mathrm{~kg}$ \\
Normal stress $\left(f_{2}\right)$ & $386 \mathrm{~N}$ (desirable) & $390 \mathrm{~N}$ (tolerable) \\
Vertical deflection & 2.7917 (tolerable) & 2.826 (tolerable) \\
$\left(f_{3}\right)$ & &
\end{tabular}

Step 11. If no target values can be chosen in $\left\{D^{\prime}-\bigcup_{p=1}^{j} G\left(\bar{X}_{p}\right)\right\}$ stop and go to Step 13, otherwise go to Step 12. In this case, other values can be chosen in Table 18 so go to Step 12 .

Step 12. Set $j=j+1$. The working groups select other target values for the goal vector $b^{j} \in\left\{D^{\prime}-\bigcup_{p=1}^{j-1} G\left(\bar{X}_{p}\right)\right\}$ and go to Step 6. Some solution results are presented in Table 19.

The second Pareto-optimal solution seems to be the best one because all the objective values are in the tolerable or desirable zone according to Table 17 . This solution can be considered satisfactory and a good compromise for all the working groups.

Step 13. The director presents the Pareto-optimal solutions to the working groups to select the best solution for everyone (stop). If other solutions are necessary go to Step 12 .

Table 20 shows the results obtained for the characteristics of the two-bar structure.

For the normal stress function, the result obtained with the IMOP approach is in the desirable zone while the solution obtained by Messac et Ismail-Yahaya [21] is in the tolerable zone according Table 17. This means that working group 2 is better satisfied with our solution. For the other functions, both results are in the same zone according Table 17. The difference between the results is that our solution is obtained by an interactive and collaborative process between the $\mathrm{DM}$ and the working groups and it is possible to generate several design scenarios (Pareto-optimal solutions) without changing the degrees of desirability. Messac and IsmailYahaya [21] provide information for only one design scenario (i.e., a single Pareto solution). If we want another solution we have to change the degrees of desirability. This IMOP algorithm has permitted to convergence to a solution that is acceptable for all the working groups. As shown, this procedure offers more flexibility for the director and his/her working groups.

\section{Conclusion}

The IMOP approach developed in this paper is an extension of the interactive nonlinear goal programming algorithm of Abdel Haleem [17]. The first contribution of the IMOP algorithm is the ability to define a reduced set of target values that can be divided into degrees of desirability to capture the working groups' preferences. This is an important contribution because it is a challenging issue in multiobjective optimization. It also subtracts the stability set from the reduced set of target values at each iteration, thus ensuring a different Pareto-optimal solution each time. Also, the distribution of the objective functions among working groups is beneficial to consider disciplinary knowledge and experience in determining the degrees of desirability. The IMOP approach generates as many new Pareto optimal solutions (design alternatives) as needed. These solutions meet as much as possible the requirements of the working groups. Also, the application of the decision that rules for choosing the target values permits the convergence to Pareto-optimal solutions in the same desirability zone (or better) for all the objectives. The approach has been successfully applied to two problems. It is true that these problems are simple but they make the application of the IMOP approach clear. In this paper, the multi-objective optimization process is centralized. Future work is also planned to use the IMOP algorithm in the case where the multi-objective optimization process is not under the control of the director but distributed to the working groups. We will be interesting by multidisciplinary optimization. Multidisciplinary optimization is a methodology used for designing complex systems that must satisfy many constraints and that must be carried out in a decentralized environment. Multidisciplinary optimization assumes a form of collaboration between the working groups because the decision variables are under the control of several working groups. The multidisciplinary optimization approaches are Concurrent subspace optimization [22-24] Bilevel integrated system synthesis [24, 25] Collaborative optimization [22, 24, 26] and Analytical Target Cascading method [27]. We are working to combine the IMOP algorithm with one of these optimization approaches.

\section{Appendices}

\section{A. Algorithm of Dauer and Krueger [19]}

We consider the classical nonlinear goal programming problem with $k$ goals (objective functions), subject $o$ a set of constraints $\{M\}$ :

$$
\begin{array}{ll}
\text { (NLGP): } & f_{1}(X) \leq b_{1} \\
& f_{2}(X) \leq b_{2} \\
& \vdots \\
& f_{k}(X) \leq b_{k} \\
\text { subject to } & M=\left\{X \in R^{n} \mid g_{r}(X) \leq 0, r=1, \ldots, m, X \geq 0\right\},
\end{array}
$$

where $X$ is the vector of decision variables $\left\{x_{1}, x_{2}, \ldots, x_{n}\right\}$ and $b_{i}, i=1, \ldots, k$ represent aspiration levels for objectives $f_{i}(X), i=1, \ldots, k$. The goals are arranged according to their priority levels, that is, if $i \leq j$ then goal $i, f_{i}(X) \leq b_{i}$ has a higher priority level than goal $j, f_{j}(X) \leq b_{j}$. It is well known 
that the fundamental premise of goal programming is that goal $i$ is sought to attain without regard to the attainability of the goals with lower priority level $j$. This idea has been used by Dauer and Krueger to develop an algorithm for solving linear, nonlinear, and integer goal programming problems. The algorithm solves $k$ singles objective function problems successively. The first and last problems are as follows:

Solving the attainment problem for goal $1, P_{1}$ is:

$$
\begin{array}{ll}
P_{1}: \quad \text { Minimize } & d_{1} \\
\text { subject to } & f_{1}(X)-d_{1} \leq b_{1} \\
& g_{r}(X) \leq 0, \quad r=1, \ldots, m \\
& d_{1} \geq 0, X \geq 0,
\end{array}
$$

where $d_{1}$ is the positive deviation for objective $f_{1}(X)$ from its goal $b_{1}$. The solution of this problem is $d_{1}^{*}$, which is the over attainment of goal 1 .

The last attainment problem for goal $k, P_{k}$ is

$$
\begin{array}{ll}
P_{k}: \quad \text { Minimize } & d_{k} \\
\text { subject to } \quad & f_{i}(X)-d_{i} \leq b_{i}, \quad 1 \leq i \leq k \\
& d_{i}=d_{i}^{*}, 1 \leq i \leq k-1 \\
& g_{r}(X) \leq 0, \quad r=1, \ldots, m, \\
& d_{k} \geq 0, X \geq 0 .
\end{array}
$$

By letting $d_{i}=x_{n+k}, i=1, \ldots, k$ the last attainment problem can be written in the form

$$
\begin{array}{ll}
P^{\prime}(G): & \text { Minimize } \\
& x_{n+k} \\
& \text { subject to } \quad g_{r}(X) \leq b_{r}, \quad r=1, \ldots, m \\
& x_{a}=x_{a}^{*}, \quad n+1 \leq a \leq n+k-1 \\
& x_{n+k} \geq 0, x_{i} \geq 0, i=1, \ldots, n,
\end{array}
$$

where $X \in R^{n+k}$ and $d_{i}^{*}, 1 \leq i \leq k-1$ is replaced by $x_{a}^{*}, n+1 \leq a \leq n+k-1$. The solution of this problem denoted by $\bar{X}=\left(\bar{x}_{1}, \bar{x}_{2}, \ldots, \bar{x}_{n+k}\right)$ is the optimal solution for the NLGP problem under consideration. Problem $P^{\prime}(G)$ can be considered as a parametric programming problem having parameters $b_{r}$ in the RHS of the constraints and can be written in the form

$$
\begin{aligned}
& P(G): \text { Minimize } f(X) \equiv x_{n+k} \\
& \text { subject to } \quad M(b)=\left\{X \in R^{n+k} \mid g_{r}(X) \leq b_{r},\right. \\
&r=1, \ldots, k+m, X \geq 0\},
\end{aligned}
$$

where $b_{r}$ is any arbitrary real number, and $x_{a}^{*}, n+1 \leq a \leq$ $n+k-1$ have been directly substituted in the inequality constraints of $P^{\prime}(G)$. The solution of problem $P(G)$ is thus the same as the solution of the $P^{\prime}(G)$ and NLGP, and the stability sets of problem $P(G)$ can be calculated.

\section{B. The Determination of the Stability Set}

Osman (1977) presented the following algorithm for the determination of the stability set of the first kind:

(1) Select an arbitrary $\bar{b} \in D$ and solve $P(G)$ to obtain $\bar{X}$ and formulate the K.K.T. conditions.

(2) Determine the values of $\bar{u}_{r}$ using any available algorithm.

(3) According to the values of $\bar{u}_{r}$, the stability set of the first kind $G(\bar{X})$ can be determined as follows:

(a) For $\bar{u}_{r}=0, r=1, \ldots, k+m, G_{1}(\bar{X})=\left\{b \mid b_{r} \geq\right.$ $\left.g_{r}(\bar{X})\right\}$

(b) For $\bar{u}_{r}>0, r=1, \ldots, k+m, G_{2}(\bar{X})=\left\{b \mid b_{r}=\right.$ $\left.g_{r}(\bar{X})\right\}$

(c) For $\bar{u}_{r}=0, r \in J \subseteq\{1, \ldots, k+m\}, \bar{u}_{r}>0, r \notin J$, $G_{J}(\bar{X})=\left\{b \mid b_{r} \geq g_{r}(\bar{X}), r \in J, b_{r}=g_{r}(\bar{X}), r \in\right.$ $J\}, G_{3}(\bar{X})=\bigcup_{\text {possible } J} G_{J}(\bar{X})$.

\section{The Sets Subtraction Algorithm [17]}

Let $b_{i}, i=1, \ldots, k$ be the elements of the universal set $V$ in the $k$ dimensional space. $V$ is considered a universal set from which some other sets $S_{i}, i=1, \ldots, I$ are subtracted. Let $V_{s}=\left\{\bigcup_{i=1}^{I} S_{i}, i=1, \ldots, I\right\}$ be the subtracted set. The elements contained in the universal set and the subtracted sets are used to determine the lower and the upper bounds for each set in each dimension. These values represent the input to the sets subtraction algorithm. Each set is represented as a record containing the lower and the upper bounds for each dimension as shown in Table 2.

The function of the algorithm is to get the difference between the universal set and the subtracted set $\left\{V-V_{s}\right\}$. This difference is defined as those elements that are contained in the set $V$ and not contained in $V_{s}$. The subtraction is done in steps. First, the algorithm gets the difference between $V$ and $V_{s}$, where $V_{s}=S_{1}$, thus getting $\left\{V-S_{1}\right\}$. Then the set $S_{2}$ is subtracted from $\left\{V-S_{1}\right\}$, thus getting $\left\{\left\{V-S_{1}\right\}-S_{2}\right\}$ and $V_{s}=\bigcup_{i=1}^{2} S_{i}$, and so on.

\section{References}

[1] R. V. Tappeta and J. E. Renaud, "Interactive multiobjective optimization procedure," AIAA Journal, vol. 37, no. 7, pp. 881-889, 1999.

[2] V. Chankong and Y. Y. Haimes, Multiobjective Decision Making: Theory and Methodology, vol. 8 of North-Holland Series in System Science and Engineering, Elsevier, New York, NY, USA, 1983.

[3] F. Mistree, O. F. Hughes, and B. Bras, Compromise Decision Support Problem and the Adaptive Linear Programming Algorithm, Structural Optimization: Status and Promise, vol. 50 of Progress in Astronautics and Aeronautics, American Institute, Washington, DC, USA, 1993. 
[4] U. Diwekar, Introduction to Applied Optimization, vol. 80, Kluwer Academic, Boston, Mass, USA, 2003.

[5] B. Suman and P. Kuman, "A survey of simulated annealing as a tool for single and multiobjective optimization," Journal of the Operational Society, vol. 57, pp. 1143-1160, 2006.

[6] A. Suppapitnarm, K. A. Seffer, and G. T. Parks, "A simulated annealing algorithm for multiobjective optimization," Engineering Optimization, vol. 33, no. 1, pp. 59-85, 2000.

[7] M. Reyes-Sierra and C. A. Coello Coello, "Multiobjective particle swarm optimizers: a survey of state-of-the-art," International Journal of Computational Intelligence Research, vol. 2, no. 3, pp. 287-308, 2006.

[8] C. A. C. Coello, D. A. VanVeldhuizen, and G. Lamonr, Evolutionary Algorithms for Solving Multi-Objective Problems, Kluwer Academic, Boston, Mass, USA, 2002.

[9] D. E. Salazar and C. M. Rocco, "Solving advanced multiobjective robust designs by means of multiple objective evolutionary algorithms (MOEA): a reliability application," Reliability Engineering and System Safety, vol. 92, no. 6, pp. 697-706, 2007.

[10] W. Gong and Z. Cai, "An improved multiobjective differential evolution based on Pareto-adaptive $\varepsilon$-dominance and orthogonal design," European Journal of Operational Research, vol. 198, no. 2, pp. 576-601, 2009.

[11] H. A. Taboada, F. Baheranwala, D. W. Coit, and N. Wattanapongsakorn, "Practical solutions for multi-objective optimization: an application to system reliability design problems," Reliability Engineering and System Safety, vol. 92, no. 3, pp. 314-322, 2007.

[12] A. Messac, S. M. Gupta, and B. Akbulut, "Linear physical programming: a new approach to multiple objective optimization," Transactions on Operational Research, vol. 8, pp. 39-59, 1996.

[13] R. V. Tappeta, J. E. Renaud, A. Messac, and G. J. Sundararaj, "Interactive physical programming: tradeoff analysis and decision making in multicriteria optimization," AIAA Journal, vol. 38, no. 5, pp. 917-926, 2000.

[14] V. Vassilev, S. C. Narula, and V. G. Gouljashki, "An interactive reference direction algorithm for solving multi-objective convex nonlinear integer programming problems," International Transactions in Operational Research, vol. 8, no. 4, pp. 367-380, 2001.

[15] R. V. Tappeta and J. E. Renaud, "Interactive multiobjective optimization design strategy for decision based design," Journal of Mechanical Design, vol. 123, no. 2, pp. 205-215, 2001.

[16] K. Miettinen and M. M. Mäkelä, "Synchronous approach in interactive multiobjective optimization," European Journal of Operational Research, vol. 170, no. 3, pp. 909-922, 2006.

[17] B. Abdel Haleem, A study on interactive multiple criteria decision making problems [Ph.D. thesis], Mechanical Design and Production Departement, Faculty of Engineering, Cairo University, 1991.

[18] A. Lamghabbar, S. Yacout, and M. S. Ouali, "Concurrent optimization of the design and manufacturing stages of product development," International Journal of Production Research, vol. 42, no. 21, pp. 4495-4512, 2004.

[19] J. P. Dauer and R. J. Krueger, "An Iterative approach to goal programming," Operational Research Quarterly, vol. 28, no. 3, pp. 671-681, 1977.

[20] M. S. A. Osman, "Characterization of the stability set of the first kind with parameters in the objective function," in Proceedings of the 10th International Conference on Mathematical Programming, Montreal, Canada, 1979.
[21] A. Messac and A. Ismail-Yahaya, "Multiobjective robust design using physical programming," Structural and Multidisciplinary Optimization, vol. 23, no. 5, pp. 357-371, 2002.

[22] J. Sobieszczanski-Sobieski and R. T. Haftka, "Multidisciplinary aerospace design optimization: survey of recent developments," Structural Optimization, vol. 14, no. 1, pp. 1-23, 1997.

[23] R. V. Tappeta, S. Nagendra, and J. E. Renaud, "Concurrent Sub-Space optimization (CSSO) MDO Algorithm in iSIGHT: validation and testing," GE research \& Development Center, 1998.

[24] I. Kroo, Distributed Multidisciplinary Design and Collaborative Optimization, VKI lecture series on Optimization Methods \& Tools for multicriteria/multidisciplinary Design, Stanford University, 2004.

[25] J. Sobieszczanski-Sobieski, D. T. Altus, M. Philips, and R. Sandusky, "Bi-level System Synthesis (BLISS) for concurrent and distributed processing," AIAA-2002-5409, American Institute of Aeronautics and Astronautics, 2002.

[26] R. D. Braun, Collaborative optimization: an architecture for large-scale decentralized design [Ph.D. thesis], Stanford University, Stanford, Calif, USA, 1996.

[27] H. M. Min, N. F. Michelena, P. Y. Papalambros, and T. Jiang, "Target cascading in optimal system design," Journal of Mechanical Design, vol. 125, no. 3, pp. 474-480, 2003. 

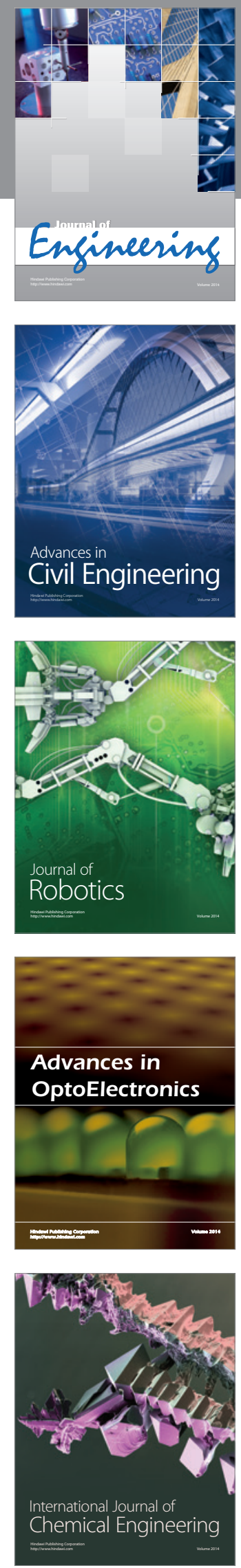

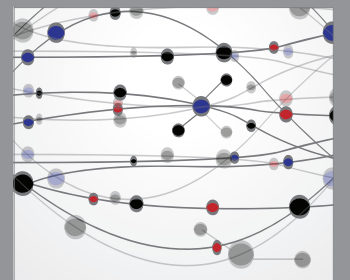

The Scientific World Journal
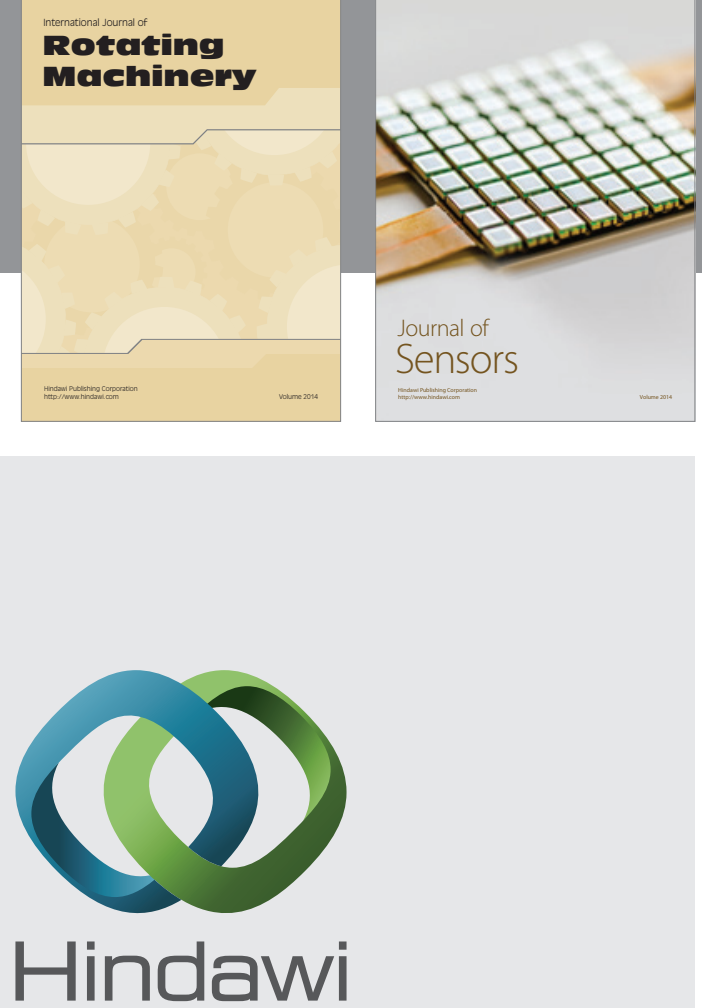

Submit your manuscripts at http://www.hindawi.com
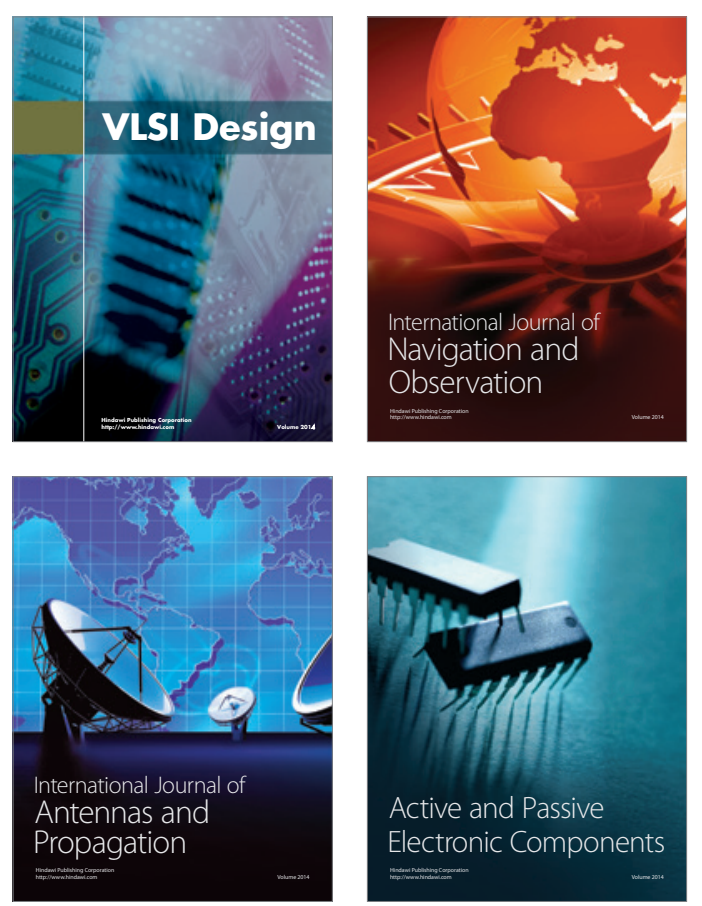
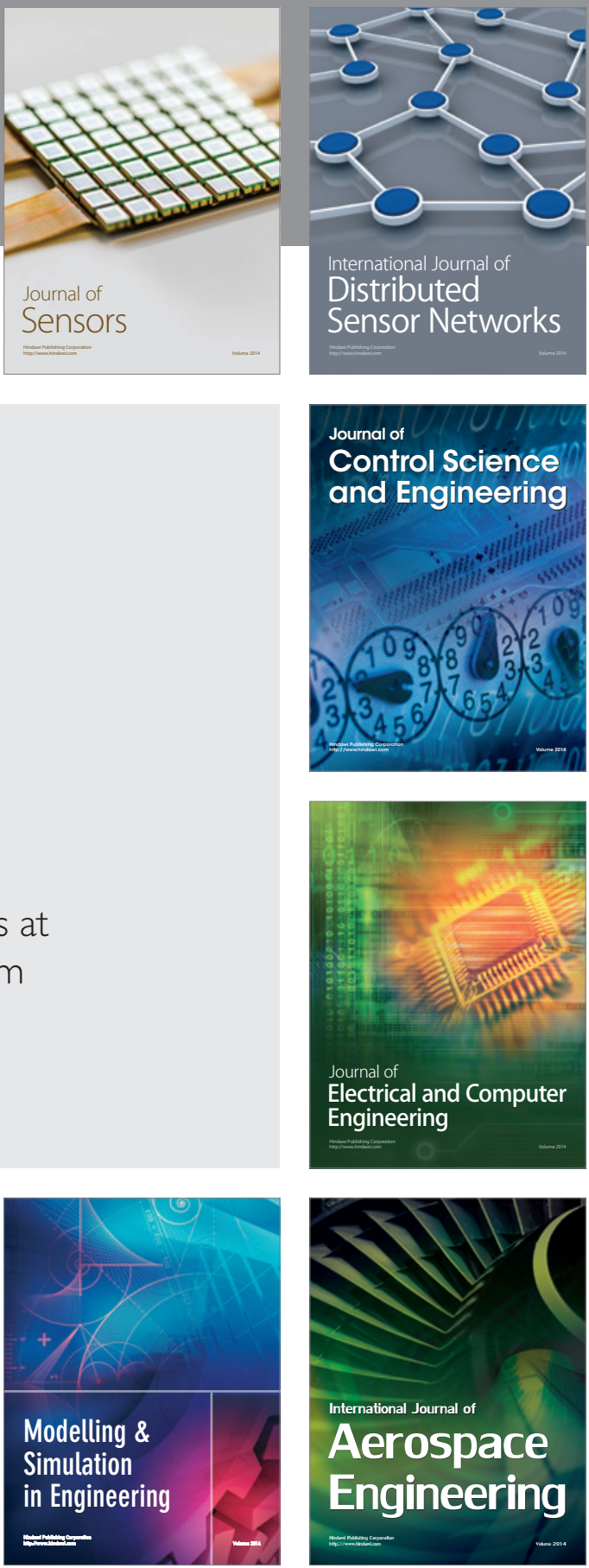

Journal of

Control Science

and Engineering
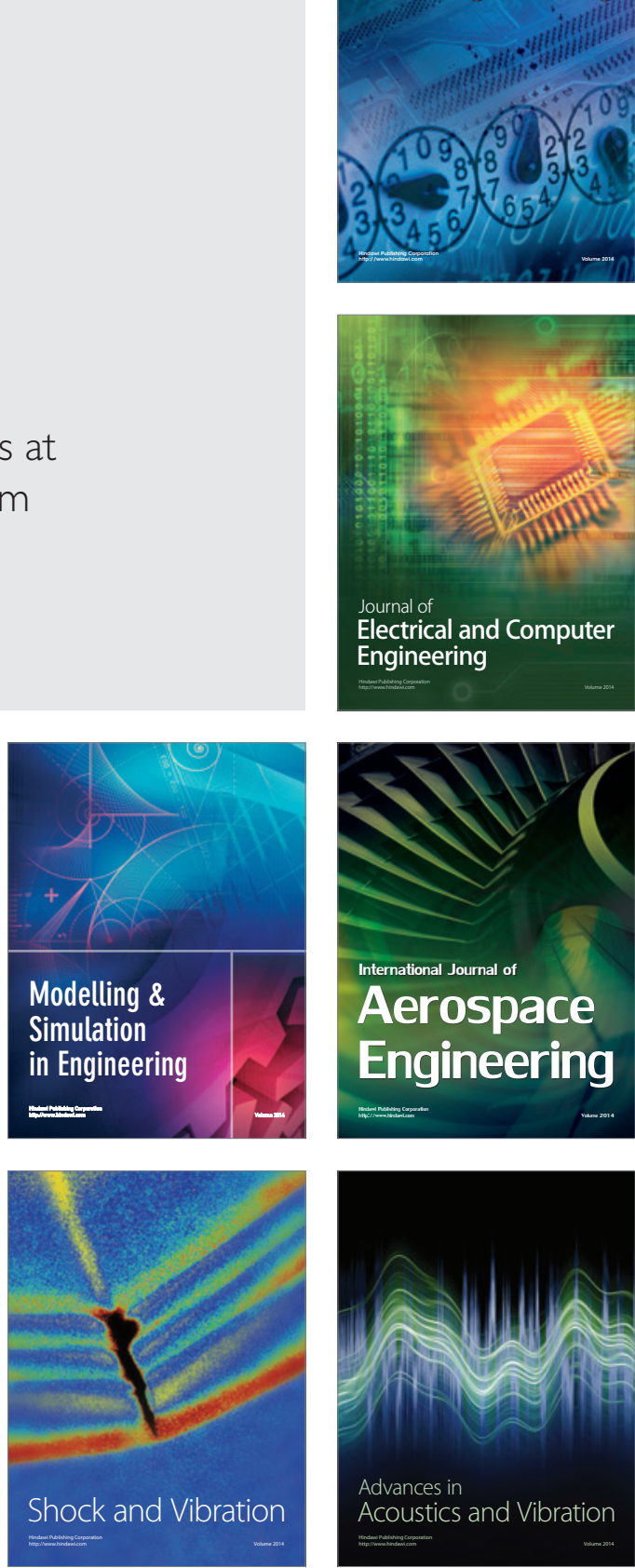